\title{
Methodological and ethical concerns associated with digital ethnography in domestic environments: participant burden and burdensome technologies.
}

\author{
Pre-published version of:
}

Nansen, B, Wilken, R, Kennedy, J, Arnold, M, and Gibbs, M. (2016) Methodological and ethical concerns associated with digital ethnography in domestic environments: participant burden and burdensome technologies. In Ethics and Visual Methods: Theory, Methodology and Practice. Eds. by Warr, D, Guillemin, M, Cox, S, and Waycott, J. New York: Palgrave Macmillan, pp. 45-60.

\begin{abstract}
This chapter reflects on methodological and ethical issues arising in a digital ethnography project conducted in domestic environments. The participatory aims of the methodological approach required participants to produce and help interpret a series of visual recordings and representations exploring their domestic digital environments. The images and videos were then uploaded using an ethnographic software application. Early in the project it became evident that researchers had limited control over important aspects of the technology, and that the technology itself was having disruptive effects in households. Further, although the study was designed to be engaging and playful for participants, the tasks of producing the data were perceived by some participants as requiring onerous levels of creativity and digital media literacy. The chapter discusses these methodological and ethical issues, and how they were largely resolved through collaborative modifications to the research design.
\end{abstract}

\section{Introduction}

This chapter reflects on some anticipated and unanticipated methodological and ethical issues that emerged during the initial waves of data collection for a project deploying mobile digital ethnographic tools and software applications within domestic environments. These issues and 
concerns arose in the context of a participatory approach that sought to encourage and empower participants to actively produce and interpret visual data that were used to explore the ways in which households adapted digital technologies in domestic environments. The production of participant-generated data was facilitated through the provision of tablets loaded with ethnographic software and prompts for visual tasks for capturing, storing, and communicating data about their household's use of broadband internet. After describing the digital ethnographic methods employed, we go on to identify and discuss the ethical concerns emerging from this process, and how these issues have been addressed.

\section{Background}

This research builds upon an established tradition in media studies of studying the 'domestication' of technologies (e.g. Haddon 2011, Silverstone and Hirsch 1992, Spigel, 2001). Historically, this area of research has examined processes of acquisition and accommodation, in which technologies are physically and symbolically located in the home. Anthropological research notes that domestication remains an ongoing process that also involves processes of dwelling, and divestment (McCracken 1988, Miller, 2012). Further, while domestication research has predominantly focused on particular technologies, such as the television (Spigel 1992), or the computer (Lally 2002), more recent research highlights the importance of studying domestic communications ecologies that increasingly involve dense interrelations of multiple technologies configuring home media environments (Nansen et al. 2009, 2011, Shepherd et al. 2007, Wilken et al. 2014).

This is because over the past decade or so, Australian households have undergone drastic transformations in the number, variety, and amount of time spent with digital media and communications technologies (ACMA 2015; Wilken et al. 2014). This study is situated within this changing household media ecology and examines high-speed broadband adoption 
and domestication. How this ongoing process of media domestication transforms the aggregated ecology of household media, and unfolds through household members interacting with particular technologies is, however, difficult to access and capture using traditional research methods. This complex sociotechnical environment calls out for sustained and close observation, yet homes are familial, intimate, and physically bounded spaces, which prove difficult for researchers to explore using in-depth qualitative ethnographic methods.

\section{Methodology, method and rationale}

Unable to observe participants in their homes for extended periods of time, researchers have opted instead to use structured interviews, surveys and focus group methods to gain insights into household technology use (e.g. OfCom 2013). Such tools are useful for gaining general insights, but less so for developing in-depth understanding of household practices. Methods that have been used to penetrate private spheres such as the home, and that might be regarded as the antecedents of the participative methods described here, have included requests for householders to descriptively diarise their daily life over time (e.g. Bolger 2003), to mark off activities on a daily time-grid or calendar (e.g. Bickman and Rog 2009), or to reconstruct their day (e.g. Kahneman 2004). Each of these methods invites research 'informants' to become research 'participants' who in effect observe themselves and reflect upon the significance of these observations.

In recent times more experimental, visual and novel applications of these forms of informant-participant data collection have been developed, the most prominent being a range of analogue media that function as 'cultural probes' to enable participants to collect, share, and negotiate data in the absence of researchers (Gaver et al. 1999). These probes involve participants taking photographs, drawing maps, keeping diaries, collecting and storing relevant materials, and so on, and have previously been used as a participatory methodology 
in domestic settings (e.g. Arnold 2004, Shepherd et al. 2007). Rather than relying on the presence and intervention of the researcher, analogue cultural probes and domestic probes are designed to encourage and empower participants to collect and interpret data themselves (Horst et al. 2004, Mackay and Ivey 2004).

In our study, we extended this participatory approach through the use of digital media by deploying iPad minis pre-loaded with a data collection software tool, EthnoCorder (http://ethnocorder.com). Using these technologies, our participants were asked to collect situated visual representations of domestic technology use. In particular we asked participants to periodically generate visual data framed around a number of playful televisual tasks. The tasks were designed to capture the household's technology use using familiar genres of a 'nature documentary', 'news report', 'paparazzi shot', 'diary room entry' and 'chat show interview'. EthnoCorder software loaded onto iPads was used to record images, video, sound and text, and to store and share the recordings with the researchers.

Methodologically, this approach was designed to include participants as collaborators in our research work. They were able to make decisions about what they thought was significant to record and share, represent their experiences and reflections using whatever medium (text, image, video) they thought appropriate, contribute to analysis of their data in subsequent interview discussions, and suggest modifications to the research design. Briefly stated, our ambitions for this method were to: (1) create a means by which households might represent the technology-related practices that occur in these intimate environments; (2) do so in a way that was open-ended, interpretive, and overtly called upon the household participants to be reflective and active; (3) create rich televisual resources that the households might use to reflect on their technology-related practices when in conversation with us; and (4) involve participants in tasks that would be playful, fun and relatively quick to complete and represented familiar televisual tropes. It was anticipated that this approach would 
heighten participants' interest and engagement in the study and diminish the burdens of involvement for participants.

This participatory method was situated within a longitudinal study also involving annual 'technology' tours and discussions with members of participating households over a period of three years. During these visits, the completed tasks, previously uploaded via the app, served as the basis for detailed discussion on participants' experiences and relationships with technologies in their homes. This paper draws on data collected during 2013-2014, the first 12 months of the study examining how high-speed broadband changes or affects the way households interact with media devices and digital technologies in the home. The sample consists of 22 households connected to the Australia Government's National Broadband Network (NBN) and represented domestic media ecologies of varying complexities, ranging from the simple (few devices with basic network setups) to the highly complex 'bleedingedge' (pushing the limits of the capabilities of technologies within their household). Households were recruited through a data sharing agreement with NBN Co who provided details of households interested in participating in research. Findings from the study analysing household media activities are available elsewhere (e.g. Arnold et al. 2014, Kennedy et al. 2015; Nansen et al. 2009, 2011, Wilken et al. 2014)..

\section{Ethics of selecting appropriate ethnographic software and designing participant tasks}

There were two stages to the development of the digital ethnographic methods used in this study: identification of a suitable device and application and the development of participant data collection tasks. EthnoCorder was selected because it offered greatest flexibility in structuring the digital tasks (see Figure 1). Like other tools, EthnoCorder users capture text, image, video and sound in response to tasks set by the research team and synch their entries to a web-based browser. A desktop application allows researchers to design and manage their 
own tasks for deployment on iOS devices. The desktop application allows for the specification of page layout, instructional text and multimedia formats of responses - for example, text, audio, image, and video. Users also have the option of adding further multimedia content to any response. Researchers are able to manage participants and data collection through an online platform, accessible only to the research team.

The features of EthnoCorder which informed our selection of the tool included the following features: relatively simple to navigate in order to complete tasks (see Figure 1); multiple users could have separate accounts on the same device enabling household members to generate and upload content independently; data are exportable as .csv spreadsheet files facilitating straightforward data management; and potential to create multiple tasks within the same application. Limitations of the EthnoCorder software include: at the time of writing it is only available on the Apple mobile operating system (iOS); it requires user action to synch data, which creates a potential barrier for researchers in accessing data if users forget this stage of the process; tasks cannot be altered remotely, which means that modifications to the research design mid-wave are problematic; and there are unlikely but potential compromises of data security as data are not encrypted when uploaded for researchers to access and EthnoCorder's cloud storage is hosted on a third-party server.

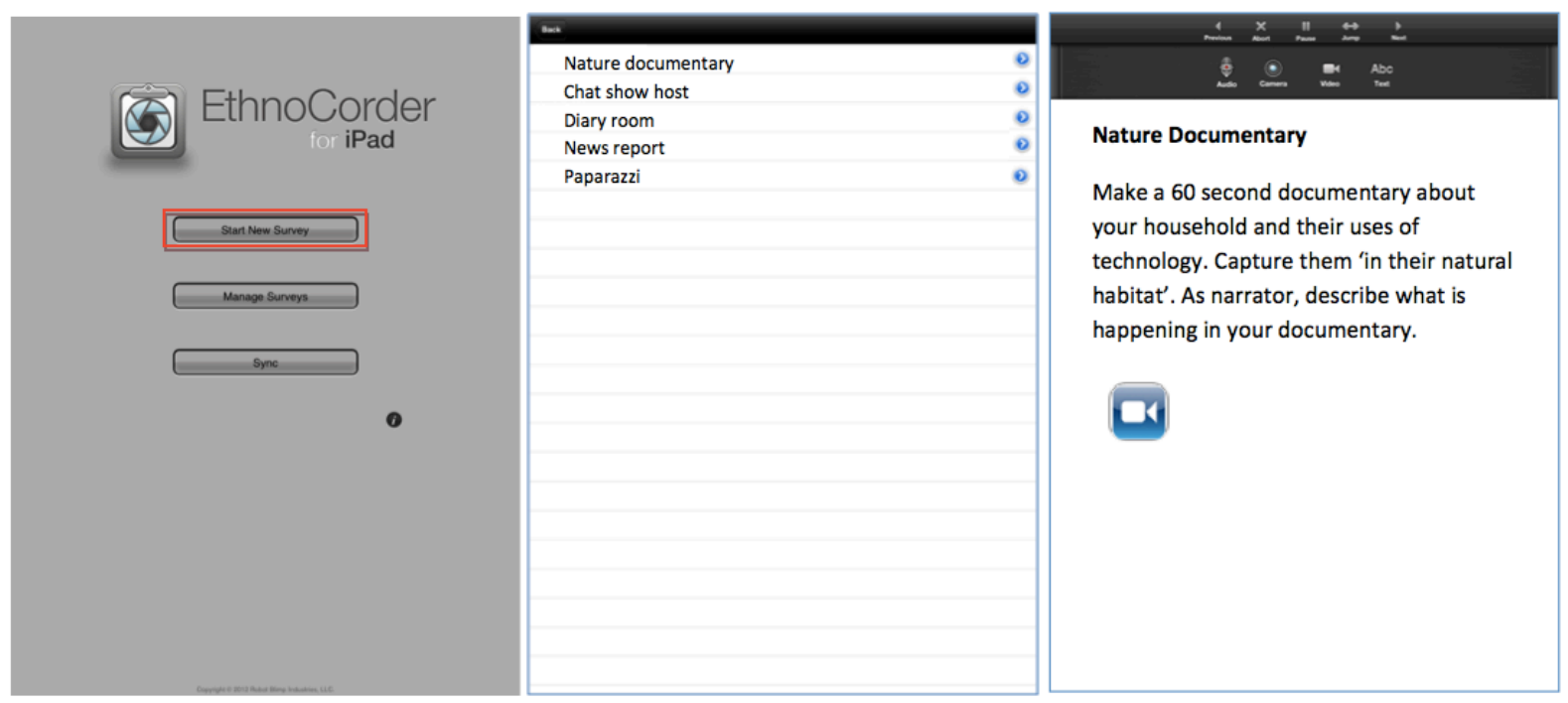

Figure 1: Screengrabs of EthnoCorder interface for iPhone/iPod Touch 
Decisions about ethnographic hardware and software are largely practical, yet they do present ethical considerations. For example, the chosen system required participants to deliberately synchronize data, rather than the system synching by default. In instrumental terms this is potentially a significant shortcoming because householders could overlook the need to synch, or may not remember how to synch. At the same time, and perhaps more interesting from methodological and ethical perspectives, this affordance provides opportunities for householders to reconsider their responses and self-edit or self-censor in ways that may compromise the value of the content as research data. The need for deliberate synching, however, means that participants must actively choose to share their data with the research team and this has ethical advantages of giving participants additional layers of control and opportunities to reconsider issues of privacy and self-presentation. The paradox is evident. Our aims in empowering participants to represent and share their experiences were to enhance their agency and control in the research process, as well as improving the quality of the data produced. Empowering participants, however, does not necessarily achieve each of these objectives. A decision about the simple affordance of manual or automated synching casts into sharp relief ethical questions raised in the negotiation of researcher-participant power differentials, where the interests of the research (in quality data) and the interests of the researched (in their own agency) may diverge.

The tasks were devised and refined through piloting and iteration over a number of research design meetings. We asked that tasks be conducted on four designated days per year at roughly three-month intervals and participating households were informed via text or email when these dates were approaching. For a 'paparazzi shot', participants were asked to take a surprise photo of a member of their household whilst using technology and then, having shown the photo to the featured household member, decide on a caption together. For the 'chat show host' we asked participants to interview a household member on their technology 
use, inquiring about their habits, routines and rituals. A 'diary room entry' required participants to speak direct to the camera explaining what they think other householders' favourite pieces of technology are and why. For a 'news report', participants were asked to create a short report about a piece of technology in their household (e.g. most loved, most frustrating, oldest, newest). For a 'nature documentary' participants were asked to make a short documentary about their household's use of technology and to capture household members 'in their natural habitat'. As with the ethnographic software, the participant data collection had ethical implications. Some tasks, either explicitly or implicitly, involved activities that meant one household member might capture footage of another householder at times when they were not aware or willing to be recorded for research purposes. Potential breaches of participants' privacy were partly addressed by building into the data collection activities the need for participants to consult with other household members about the footage captured, for example, deciding on a caption to go with an image. This gave relevant household members opportunities to review recordings and potentially veto footage being uploaded for researchers to access and use as research data.

\section{Findings and reflections on ethics of using digital ethnography tools}

On the first household visit, we set aside some time with the household to set up their new iPad mini, register the app to the user, and go through the televisual tasks. Only a few of the participants indicated unfamiliarity with the features of the iPad mini device, though many were not sure of how to set up the device and install gifted applications. Participants were generally enthusiastic about completing the research tasks and some expressed initial thoughts about what they might capture when it came time to complete them at set intervals over the longitudinal study. Over the course of 2013-2014 the first cohort of participants were asked to choose and complete four of the six preinstalled tasks, and then upload their 
recordings using the EthnoCorder application. Around half of the participating households completed the four tasks in the initial wave of data collection. Although households could choose any of the tasks, the 'paparazzi shot' and 'news story' tasks proved to be the most popular. Only a few participants opted to complete a 'nature documentary', 'diary room entry', or 'chat show host' tasks in these initial stages of data collection.

Prior to the second wave of interviews with household members, we speculated that these preferences might have been due to some scenarios being perceived as being more time consuming than others, requiring additional creative input from participants, more challenging to complete to participants' satisfaction, or a combination of these factors. In discussions with participants in the second household visits, interviews and technology tours, we asked about perceptions of the iPad device, the usability of the EthnoCorder program and the tasks, in an effort to identify potential obstacles to completing the tasks, or not selecting particular kinds of tasks. These discussions revealed a range of reasons for participants not completing tasks and their responses; these are divided into categories of household priorities or technical issues, and are described below. This discussion is followed by some reflections on the methodological and ethical implications of the perceived obstacles that were associated with the tasks.

\section{Household priorities}

In making our requests we were asking for the expenditure of what for many are scarce resources - time and energy. As might be expected, many participants spoke about the research tasks conflicting with household circumstances, such as lifestyle and work commitments, busy family routines and demanding work schedules, and holidays. In particular, such everyday constraints and pressures meant that requests to complete tasks were easily overlooked and forgotten, or were always on the "to-do" list but pushed back to a 
point where they never came to the top of the list. On occasion, they were perceived as relatively unrewarding chores or work-tasks that could be dispensed with to make time to attend to other demands.

In addition to these readily anticipated obstacles, we also found issues to do with the personal, self-imposed demands of completing a creative task. In one sense, the open-ended tasks, such as those we set for participants to complete, presented no particular hurdles. We were keen to affirm that there were no entry barriers, that nothing was too short, that there were no right or wrong responses and that all representations of experience and attitude were of value so far as we are concerned. However, these assurances did not necessarily dispel the self-imposed expectations of some participants to be creative, reflective and expressive, nor did they ensure that participants felt comfortable about how they would be perceived while being audio-recorded or videoed, and trying to anticipate and meet the imagined expectations of researchers.

We anticipated the time-scarcity experienced by many households, but underestimated the 'tyranny of the blank page' experienced by many authors, including some of our participants. Our attempts to alleviate potential burdens of involvement in the research, by designing research activities that were oriented toward familiar media forms and which were intended to be quick and amusing to produce,, proved to be only partially successful. This raises some questions about the assumptions of participatory approaches in qualitative and ethnographic research, which may be seen as more inclusive and empowering than more structured methods, but which can place increased demands on the ways in which participants are obliged to 'participate' in research. In some circumstances, opportunities for creative contributions may not necessarily be welcomed or valued in relation to standard research methods, such as interviews and surveys. Clearly, there is a balance to be struck with methodological familiarity and convenience - one that we have found is often resolved in 
early and clear discussions with participants about the objectives and approach of the research.

\section{Technical Obstacles}

In addition to the range of personal issues moderating participants' capacity to undertake the televisual tasks, we also found that our participating households encountered a range of technical 'reverse salients' (Hughes 1983). In the language of science and technology studies, this refers to weak links in the chains of networked hardware and software that significantly compromise the capacity of the technology as a whole to function as intended. Primarily, these reverse salients centred on complications experienced with the ethnographic software application rather than the iPad hardware. In particular, during the time period since the initial visit, explanation and hand-over of the tablet and software, a number of updates were made to the app, initiated by Apple's changes to its operating system. This left us relying on participants themselves to update the app. In many devices, a failure to update caused errors in the app and led it to crash. In second-wave interviews, these technical difficulties were reported by participants as frustrating and disappointing. More common, however, were frustrations arising from some participants' limited digital media competency or confidence in using the tablet, operating system or app. This meant that, for some participants, the research-related technologies proved difficult to use and were not easily integrated into their established domestic media ecology and routines.

The personal and technical difficulties encountered by research participants are of methodological concern in so far as they undermine the quality and quantity of data produced. Of further concern were the ethical implications that are associated with the difficulties that were experienced. 


\section{Ethical Implications of household priorities}

Participant burden in terms of demands on time in an age where many individuals perceive themselves to be time poor is itself an ethical concern. In effect we were asking individuals to make a personal sacrifice for the collective good, and the ethics of that trade-off needs careful consideration. This is partly addressed in the mandated processes of acquiring ethical approval to conduct research where, among many other things, university, and other institutional, ethics approval processes require researchers to argue to the satisfaction of the committee that the research outcomes generated through the proposed research outweigh the burden of involvement in research for participants.

A number of arguments might be made to support the judgement that in our case the participant burden is outweighed by the benefits of the research. The outputs have been published in peer-reviewed journals and have been assessed as significant. The participants gave informed consent to volunteer in the study and benefited in receiving iPads as a gift. Some participants acquired improved digital media skills, and some households and participants benefited from the opportunity to reflect on their technology use. Some participants responded to suggestions to complete the tasks communally, and experienced these activities as enjoyable. In these circumstances, the time expenditure contributed to the study could be regarded as contributing to the convivial sociality of the household. However, those who are not researchers may well come to a different conclusion when balancing the value of research outputs against the sacrifices of time and effort made to obtain research inputs.

Similarly, the frustration some participants experienced in meeting the challenge implicit in the creative nature of the task raised both methodological and ethical concerns. 
The tasks were intended to be open-ended rather than prescriptive, and inclusive rather than exclusionary. Some participants, however, interpreted the tasks as requiring a degree of creative professionalism and polish that they did not feel they possessed, or a degree of reflective thoughtfulness and profundity that they could not deliver. In short, they felt that they were not up to the creative and communicative challenges, and this raises ethical concerns that some participants might be left feeling inadequate or even as having 'failed'.

\section{Ethical Implications of technical obstacles}

As noted, the technical obstacles we observed may have had the unintended consequence of excluding those without the requisite expertise to participate. This outcome raises methodological and ethical concerns. The key reverse salient occurred as a consequence of operating system updates, posing a particular problem for Apple app developers, but which raises a more generally applicable ethical concern. In particular, the deployment by the researchers of the EthnoCorder app, the iOS operating system, the iPad and other actors in the network, constitutes the intervention of third parties standing between the researcher and the participants. Operating system updates were out of the control of the participants and the researchers. In effect the researchers were outsourcing important aspects of the relationship between themselves and the participants to others, and were beholden to third parties for the conduct of important aspects of the research.

A further ethical consideration associated with the technology emerged through the implications of our intervention in the household's domestic media ecology. Providing the household with an iPad is problematic, particularly in the context of social research directly related to technology appropriation and practices. In the data collected through these early stages of the longitudinal study there is an indication that the disruptive qualities of the technology, particularly the tools used to deliver the tasks - the iPad minis — intervened in 
and disrupted the very thing we were studying - the established household media ecology and routines of technology use.

This technological intervention raised other ethical issues. In requiring participants to spend more time engaging with electronic devices it may be exacerbating the potential risks associated with this. For example, it has been argued that over-use of technologies such as iPads can cause significant neurological harms (Greenfield 2014). Whilst such claims are far from proven, the iPad may not be without social or psychological harm, and it may be admitted that we have no specific mechanisms in place to trace or respond to any harms that might arise from the iPad's presence.

In summary, a number of ethical concerns emerged from both the research design and data collection process. These related to the relatively high participant burden, issues of perceived capacity and confidence required to complete the tasks, and the disruptive qualities of the device. This research shows that visual methods and video data are not unproblematic, though they have sometimes been represented as such in discussions of household technology methods elsewhere (Blythe et al. 2002, Petersen 2002).

Many of the household and technical ethical concerns that were identified were subsequently addressed through modifications to the research design, and in collaboration with the research participants. These modifications included simplifying the data collection activities and burden of choice by setting specific tasks from the available list for each period of data collection. While these strategies in some ways undermined the aims of the participatory methodology by giving more structure to the activities, they also served to make the process more inclusive in enabling more participants to feel able to contribute to the study. Rather than relying only on the iPad minis and the EthnoCorder app, participants were able to use their own digital devices or software to complete tasks. Participants were also able to use email to communicate with researchers and send data. 


\section{Conclusion}

In summary, digital ethnography tools offer opportunities for visual ethnographic research and participatory studies. Yet, these tools, may become obstacles to data collection and introduce new, and unanticipated, methodological and ethical concerns. The tasks invited people to actively participate in the research in a meaningful way, but this also imposes upon the daily lives of participants. The open-ended and expressive tasks also resulted in selfimposed standards that potentially constrained the ways in which some participants felt they could contribute to the project. A lack of familiarity with new technologies asks for patience from the participants and researchers, and may add to the burdens of participation. Important aspects of the research process may not be in the control of the researchers. Finally, the methodology entails the introduction of a technology into domestic environments, and harms that might be associated with this warrant consideration.

Using digital ethnography tools can add further layers of methodological and ethical complexity to data collection processes. There is, however, potential for such tools to provide opportunities to engage with participants in a situated manner over extended periods of time in ways that would otherwise be difficult to achieve. Moreover, the digital ethnography approach offers important opportunities for realising the methodological ambitions of cultural probe approaches by intervening in and disrupting the daily flow of life in order to prompt reflective engagement with familiar, everyday environments. Our research to date in this longitudinal project suggests that, if carefully and thoughtfully managed — in consultative, flexible, and iterative ways - these kinds of ethnographic tools can strengthen participant engagement and commitment to the research, as well as build participant-researcher relationships, and yield rich, and otherwise hard to obtain, participant insight, and selfreflection. 


\section{Acknowledgements}

This work was supported by the Australian Research Council [DP130101519]. The authors would like to thank the research participants for their enthusiasm, time and support. Participant permission has been granted for the use of images in this publication.

\section{References}

Australian Communications and Media Authority (ACMA). 2015 Communications Report. Canberra: ACMA.

Arnold, Michael, Thomas Apperley, Bjorn Nansen, Rowan Wilken, and Martin Gibbs. 2014. "Patchwork Network: Spectrum Politics, the Digital Home and Installation of the Australian National Broadband Network". In Management of Broadband Technology Innovation: Policy, Deployment and Use, edited by Choudrie, Jyoti and Catherine Middleton C, 25-42. New York: Routledge.

Arnold, Michael. 2004. "The Connected Homes Project: Probing the Effects and Affects of Domesticated ICTs.” In Artful Integration: Interweaving Media, Materials and Practices, Vol. 2. Proceedings of the Eighth Biennial Participatory Design Conference, edited by A. Bond. Toronto.

Bezemer, Jeff, and Diane Mavers. 2011. "Multimodal Transcription as Academic Practice: A Social Semiotic Perspective.” International Journal of Social Research Methodology 14(3): 191-206.

Bickman, Leonard, and Debra Rog. 2009. The SAGE Handbook of Applied Social Research Methods. Thousand Oaks, CA: SAGE Publications, Inc. 
Blythe, Mark, Andrew Monk, and Jisoo Park. 2002. “Technology Biographies: Field Study Techniques for Home Use Product Development.” In Proceedings of CHI 2002: Changing The World, Changing Ourselves. Minneapolis: 658-659.

Bolger Niall, Angelina Davis, and Eshkol Rafaeli. 2003. "Diary Methods: Capturing Life as it is Lived." Annual Review of Psychology 54: 579-616.

Cox, Susan, Sarah Drew, Marilys Guillemin, Catherine Howell, Deborah Warr, and Jenny Waycott. 2014. Guidelines for Ethical Visual Research Methods. The University of Melbourne, Melbourne.

Gaver, Bill, Tony Dunne, and Elena Pacenti. 1999. "Cultural Probes.” Interactions (February): 21-29.

Goldman, Ricki. 2009. "Video representations and the Perspectivity Framework." In Video Research In The Learning Sciences, edited by Goldman, Ricky, Roy Pea, Brigid Barron and Sharon J. Derry, 3-38. New York: Routledge.

Greenfield, Susan. 2014. Mind Change: How Digital Technologies Are Leaving Their Mark on Our Brains. Rider.

Gubrium, Aline, Amy Hill, and Sarah Flicker. 2013. "A Situated Practice of Ethics for Visual and Digital Methods in Public Health Research and Practice: A Focus on Digital Storytelling." American Journal of Public Health: e1-e9.

Haddon, Leslie. 2011. "Domestication Analysis, Objects of Study, and the Centrality of Technologies in Everyday Life.” Canadian Journal of Communication 36: 311-323.

Horst, Willem, Ties Bunt, Stephan Wensveen, and Lisa Cherian. 2004. "Designing probes for empathy with families." In Proc. Dutch Directions in HCI'07. NY: ACM Press: 29 37.

Hughes, Thomas P. 1983. Networks of Power: Electrification in Western Society, 1880-1930. The Johns Hopkins University Press. 
Jewitt, Carey. 2012. An Introduction to Using Video for Research. NCRM Working Paper: NCRM.

Kahneman, Daniel, Alan Kreuger, David A. Schkade, Norbert Schwarz, and Arthur A. Stone. 2004. “A Survey Method for Characterizing Daily Life Experience: The Day Reconstruction Method”. Science 306: 1776-1780.

Kennedy, Jenny, Michael Arnold, Bjorn Nansen, Rowan Wilken, and Martin Gibbs. 2015. "Digital Housekeepers and Digital Expertise an the Networked Home." Convergence: The International Journal of Research into New Media Technologies. Advanced online publication DOI:10.1177/1354856515579848.

Lally, Elaine. 2002. At Home with Computers. Oxford: Berg.

Mackay, Hugh, and Darren Ivey. 2004. Modern Media in the Home: An Ethnographic Study. Rome, Italy: John Libbey.

McCracken, Grant. 1988. Culture and Consumption. Bloomington: Indiana University Press.

Miller, Daniel. 2012. Consumption and its Consequences. Cambridge: Polity. Nansen, Bjorn, Michael Arnold, Martin Gibbs, and Hilary Davis. 2009. "Domestic Orchestration: Rhythms in the Mediated Home." Time and Society 18(2): 181-207. Nansen, Bjorn, Michael Arnold, Martin Gibbs, and Hilary Davis. 2011. "Dwelling with Media Stuff: Latencies of Materiality in Four Australian Homes." Environment and Planning D 29(4): 693-715.

OfCom. 2013. Children and Parents: Media Use and Attitudes Report. London: OfCom. Petersen, Marianne Graves. 2002. Designing for Learning in Use of Everyday Artefacts. (PhD Thesis, University of Aarhus, 2002). Aarhus: University of Aarhus.

Shepherd, Chris. Michael Arnold, Craig Bellamy, and Martin Gibbs. 2007. "The Material Ecologies of Domestic ICTs.” The Electronic Journal of Communication 17(1-2). 
Silverstone, Roger, and Eric Hirsch (Eds). 1992. Consuming Technologies: Media And Information In Domestic Spaces. London: Routledge.

Snell, Julia. 2011. "Interrogating video data: systematic quantitative analysis versus micro ethnographic analysis.” International Journal of Social Research Methodology 14(3): $253-258$.

Spigel, Lynn. 1992. Make Room for TV: Television and the Family Ideal in Postwar America. Chicago: University of Chicago Press.

Spigel, Lynn. 2001. "Media Homes: Then and Now.” International Journal of Cultural Studies 4(4): 385-411.

Wilken, Rowan, Michael Arnold, Bjorn Nansen, Jenny Kennedy, and Martin Gibbs. 2014. "National, Local and Household Media Ecologies: The Case of Australia's National Broadband Network." Communication, Politics and Culture 46(2): 136-154. 